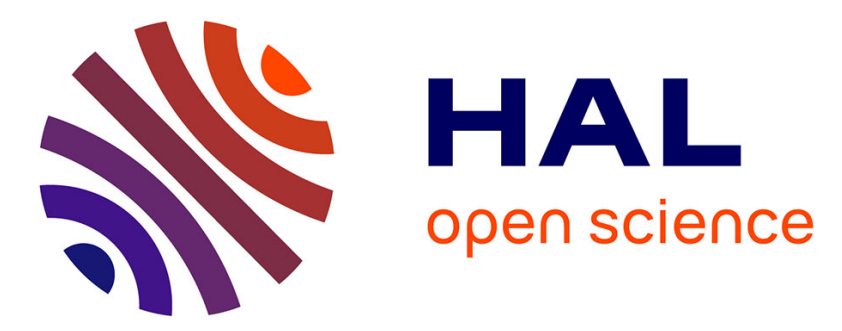

\title{
Polarimetric interferometer for nanoscale positioning applications
}

\author{
Suan Xu, Karim Ouedraogo, Luc Chassagne, Suat Topsu, Patrick Juncar, \\ Yasser Alayli
}

\section{- To cite this version:}

Suan Xu, Karim Ouedraogo, Luc Chassagne, Suat Topsu, Patrick Juncar, et al.. Polarimetric interferometer for nanoscale positioning applications. Review of Scientific Instruments, 2008, 79, pp.125104. 10.1063/1.3053224 . hal-00830878

\section{HAL Id: hal-00830878 https://hal.science/hal-00830878}

Submitted on 5 Jun 2013

HAL is a multi-disciplinary open access archive for the deposit and dissemination of scientific research documents, whether they are published or not. The documents may come from teaching and research institutions in France or abroad, or from public or private research centers.
L'archive ouverte pluridisciplinaire HAL, est destinée au dépôt et à la diffusion de documents scientifiques de niveau recherche, publiés ou non, émanant des établissements d'enseignement et de recherche français ou étrangers, des laboratoires publics ou privés. 


\title{
Polarimetric interferometer for nanoscale positioning applications
}

\author{
S. Xu, K. Ouedraogo, L. Chassagne, S. Topçu, P. Juncar, and Y. Alayli \\ LISV, University of Versailles Saint-Quentin, PRES UniverSud, Versailles, France LNE-INM/Cnam rue du \\ Landy, Seine Saint Denis, France
}

(Received 29 July 2008; accepted 27 November 2008; published online 22 December 2008)

\begin{abstract}
We propose and demonstrate a displacement control method at the subnanometric scale based on a Michelson interferometer combined with a polarimeter and a phase-locked loop electronic board. Step by step displacements with a step value of $5 \mathrm{~nm}$ are presented. A repeatability of $0.47 \mathrm{~nm}$ is obtained from back and forth displacements over $1 \mu \mathrm{m}$ range. We show that a residual ellipticity of less than $10^{\circ}$ on the polarization state leads to a positioning error of less than $1 \mathrm{~nm}$. Such system could be used over millimeter range displacements in a controlled surrounding environment leading to numerous applications in nanometrology. (C) 2008 American Institute of Physics.
\end{abstract}

[DOI: $10.1063 / 1.3053224]$

\section{INTRODUCTION}

Development in industry is asking for improved resolution and higher accuracy in dimensional metrology. With the miniaturization of electronic components, the need for subnanometer accuracy in dimensional metrology is increasing. Only two kinds of sensors allow reaching nanometer scale accuracy over macroscopic displacement ranges: laser interferometer and linear encoder. Capacitive and inductive sensors have a measurement range of only some micrometers. The linear encoders operate with no mechanical contact between a scanning head and a scale tape composed of a diffraction grating. Their resolution is limited by the periodicity of the gratings to tens of nanometers. A subnanometric accuracy seems hard to reach with this kind of sensors because the gratings of the head and the scale tape are manufactured by means of photolithography. Photolithography is the process in which patterns, specific for a particular chip design, are projected onto resist on silicon wafers. This is done in a wafer stepper or scanner. A semiconductor device is built up from several layers, which need to be positioned onto each other. After each layer the wafer is extracted from the wafer scanner and etched. For the next illumination the wafer has to be repositioned, and in order to form a connection of the lines in two different layers a certain overlay has to be guaranteed. The position information for this is gathered with the use of laser interferometer systems. By miniaturization of integrated circuits the energy consumption can be reduced and the speed of chips can be increased. At this moment linewidths of $80 \mathrm{~nm}$ are in use for volume production of advanced memory devices. The overlay error should typically be $30 \%$ of the linewidth. ${ }^{1-3}$ Since the laser interferometer is only one component in the entire system the uncertainty of this system has to decrease below the nanometer region. Current demands on the laser interferometer system are already in the subnanometer region. The requirements for future lithography technology are presented by the ITRS roadmap (International Technology Roadmap for Semiconductors). ${ }^{4}$ As one of the difficult challenges the metrology and defect inspection of linewidths below $45 \mathrm{~nm}$ is mentioned through 2009. Beyond 2009 the challenge becomes even bigger for measuring critical dimensions down to $7.2 \mathrm{~nm}$ in 2018.

To improve the accuracy of displacement laser interferometer systems toward the subnanometer level, research has to be conducted in order to investigate the error sources at this level and to develop a subnanometric position control method. ${ }^{5-9}$ This paper describes the optical sensor we developed in order to control the position of a moving stage with an accuracy at the subnanometric scale. Our system is mainly dedicated to microelectronic fabrication process and nearfield scanning microscopy where most applications need millimeter scale displacements. Under very strict control of the room parameters (temperature, mechanical vibrations, etc.), the method could be used beyond millimeter displacement ranges.

\section{THE POLARIMETRIC INTERFEROMETER}

Our position sensor is a combination of a homodyne laser interferometer and a polarimeter, as shown in Fig. 1. The polarization state of the laser source is controlled at the input of the interferometer, thanks to a Glan-Thompson polarizer combined with a large bandwidth half-wave plate. Such system allows us to polarize linearly the laser beam and to adjust the polarization plan at $45^{\circ}$ with regard to the propagation plan. The Michelson interferometer is composed of a polarization beam splitter and three quarter-wave plates put on each arm of the interferometer and at the output of the system. The main difference with a conventional laser interferometer is that the common photodetector used in this kind of sensor is replaced by an optical polarimeter. The polarimeter determines the ellipsometric parameters $(\psi, \varepsilon)$ that characterize the polarization state of the laser beam. The parameters $\psi$ and $\varepsilon$ represent, respectively, the azimuth and the ellipticity of the polarization state of the light beam. In our method, the displacement of the moving mirror is linked to the azimuth $\psi$ instead of the phase of the interferogram. As shown in Sec. II A, this allows a more accurate position con- 


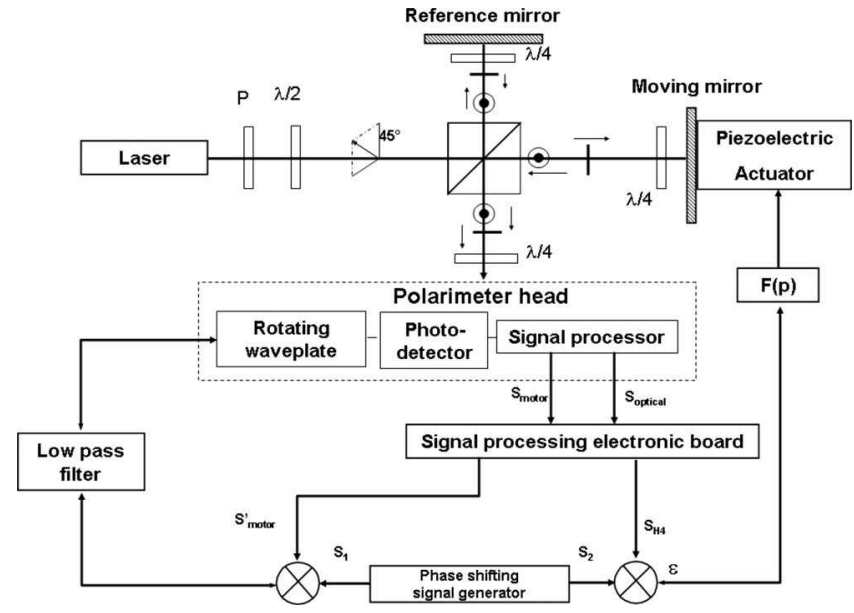

FIG. 1. Principle of the polarimetric laser interferometer for a subnanometric position control moving stage.

trol of the moving mirror. The position servo loop control of the moving mirror, also reported on Fig. 1, is discussed in detail in Sec. II B.

\section{A. Principle}

If we refer to $E_{s}$ and $E_{p}$ as the perpendicular and parallel components of the laser field to the propagation plane, using the Jones matrix calculus, ${ }^{10-12}$ the $s$ - and $p$-components of the optical field at the output of the interferometer are (the temporal and spatial propagation terms are omitted)

$$
\begin{aligned}
& \overrightarrow{E_{p}}=\frac{1}{\sqrt{2}}\left(\begin{array}{l}
1 \\
-i
\end{array}\right), \\
& \overrightarrow{E_{s}}=\frac{1}{\sqrt{2}} \exp ^{( \pm i \phi)}\left(\begin{array}{l}
1 \\
+i
\end{array}\right) .
\end{aligned}
$$

Thus, at the output of the interferometer, we have two circularly polarized beams with a phase difference equal to $\pm \phi$ depending on the value and on the sense of the displacement. The polarimeter combines the two beams leading to an electromagnetic field with a linearly polarized state given by

$$
\vec{E}=\overrightarrow{E_{p}}+\overrightarrow{E_{s}}=\left(\begin{array}{l}
\cos (\phi) \\
\mp \sin (\phi)
\end{array}\right)=\left(\begin{array}{c}
\cos \psi \\
\sin \psi
\end{array}\right) .
$$

Equation (3) allows us to link the displacement of the mirror to the polarimetric angle $\psi$ by

$$
\Delta x=\frac{\lambda_{0} \psi}{2 \pi n},
$$

where $\lambda_{0}$ is the wavelength under vacuum of the laser source and $n$ is the refractive index of air.

The polarization state of the light beam could also be defined by the Stokes parameters. ${ }^{13-15}$ For a normalized and a polarized laser beam, the Stokes parameters are given by

$$
\begin{aligned}
& S_{0}=1, \\
& S_{1}=\cos 2 \varepsilon \cos 2 \psi, \\
& S_{2}=\cos 2 \varepsilon \sin 2 \psi,
\end{aligned}
$$

$$
S_{3}=\sin 2 \varepsilon .
$$

The value of $\psi$ is then equal to

$$
\psi=\frac{1}{2} \arctan \left[\frac{S_{2}}{S_{1}}\right] .
$$

In our case, the Stokes parameters are measured with a spinning quarter-wave plate polarimeter. This system uses a mechanically rotated quarter-wave plate and employs Fourier analysis of the detected signal to calculate the ellipsometric angles $\varepsilon$ and $\psi$ from the amplitude and the phase of the second $(2 f)$ and fourth $(4 f)$ harmonics of the signal. The intensity measured by the photodetector is a function of the rotative speed of the quarter-wave plate $(\omega)$ and the Stokes parameters $\left(S_{1}, S_{2}, S_{3}\right.$, and $\left.S_{4}\right)$ of the incoming light beam. It is equal to ${ }^{16}$

$$
\begin{aligned}
I(\omega)= & \frac{1}{2}\left[\left(S_{0}+\frac{S_{1}}{2}\right)+\frac{S_{1}}{2} \cos (4 \omega t)+\frac{S_{2}}{2} \sin (4 \omega t)\right. \\
& \left.-S_{3} \sin (2 \omega t)\right] .
\end{aligned}
$$

By carrying out a Fourier analysis on the intensity $I(\omega)$, we find that

$$
\begin{aligned}
& \frac{S_{1}}{2}=\frac{2}{\pi} \int_{0}^{2 \pi} I(\omega t) \cos 4 \omega t d t, \\
& \frac{S_{2}}{2}=\frac{2}{\pi} \int_{0}^{2 \pi} I(\omega t) \sin 4 \omega t d t,
\end{aligned}
$$

which are, respectively, the real and the imaginary parts of the fourth harmonic of $I(\omega)$. If $\Delta \varphi$ represents the phase shift of the $4 f$ harmonic component of $I(\omega)$, by calculating the term $\arctan \left[S_{2} / S_{1}\right]$ and using Eq. (6), we find that

$$
\psi=\frac{1}{2} \Delta \varphi
$$

Hence, combining Eqs. (4) and (9), we deduce a relation between the displacements of the target mirror $\Delta x$ and the phase shift $\Delta \varphi$ of the fourth harmonic component of $I(\omega)$ as

$$
\Delta x=\frac{\lambda_{0} \Delta \varphi}{4 \pi n} .
$$

Hence, we can control the position of the moving mirror by controlling the phase $\Delta \varphi$. According to Eq. (10), a displacement of the moving mirror of $\lambda_{0} / 2 n$ corresponds to a phase shift on the fourth harmonic of $2 \pi$. Using commercially available polarimeters (PA450 Thorlabs) with a resolution on $\psi$ values of $0.01^{\circ}$, the intrinsic limit of this position control method is $70 \mathrm{pm}$.

\section{B. The servo loop of the position control}

The phase-locked loop control method is described in Fig. 2. A digital synthesis generator puts out two synchronous transistor-transistor logic (TTL) signals $S_{1}$ and $S_{2}$ at the same frequency of $14 \mathrm{~Hz}$. The generator is able to make phase shifts on both signals. The signals $S_{\text {motor }}$ and $S_{\text {optical }}$ are both issued from the polarimeter head. $S_{\text {motor }}$ contains information about the rotation speed $\omega$ of the rotating motor. As seen in Eq. (7), $S_{\text {optical }}$ is made up of dc, $2 f$, and $4 f$ compo- 


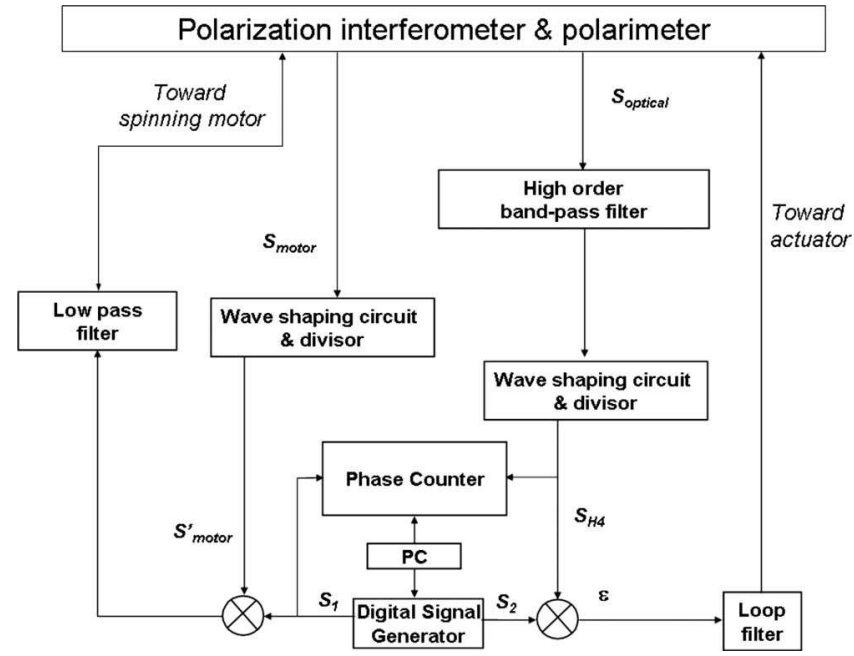

FIG. 2. Experimental setup. The output signal of the polarimeter is filtered by an electronic board in order to separate second and fourth harmonics. The phase of the fourth harmonic is compared to the phase reference issued from a digital signal generator. The motor of the spinning quarter-wave plate is also locked on the same reference.

nents. At the output of the signal processing electronic board, $S_{\text {motor }}$ and the $4 f$ component of $S_{\text {optical }}$ are converted to a TTL signal, thanks to a wave shaping electronic board. $S_{\text {motor }}$ is phase compared to $S_{1}$ in order to control the angular speed $\omega$ of the motor by a phase-lock loop system made with a phase comparator, a low-pass filter, and the electronic command of the rotating motor. The frequencies of the $2 f$ and $4 f$ components are equal to, respectively, 56 and $112 \mathrm{~Hz}$. The $4 f$ component is filtered with an eighth order continuous-time active filter and is divided by 8 in frequency $\left(S_{H 4}\right)$. Then the phases of both $S_{H 4}$ and $S_{2}$ are compared. The position of the moving mirror is controlled, thanks to the phase of $S_{2}$. The loop filter is a proportional-integral control block with adjustable parameters. If a phase shift occurs on $S_{2}$, the signal error $\varepsilon$ becomes non-null and the servo controller moves the target mirror in order to compensate this phase shift using the Doppler effect. Since the phase shift can be quantified according to Eq. (10), the displacement of the translation stage could be made step by step with a very accurately known step value. The intrinsic accuracy of the step value is limited by the knowledge of the laser wavelength $\left(1 \sigma=10^{-9}\right),{ }^{17,18}$ and by the refractive index of air if the measurements are not made under vacuum. The displacement ranges could reach several centimeters depending on the translation stage used in the system.

\section{Experimental setup and results}

The polarimeter used in our prototype is a spinning quarter-wave plate polarimeter (PA450, Thorlabs). The rotating motor has a rotation frequency of $28 \mathrm{~Hz}$. The digital synthesis generator is a AFG3102 Tektronix model and the phase meter is an HP53132A from Hewlett Packard. In our system, the polarimeter is used not only for the positioning control but also to visualize the polarization state of the laser beam at the output of the interferometer.

As seen in Eq. (10), the displacement value $\Delta x$ is related

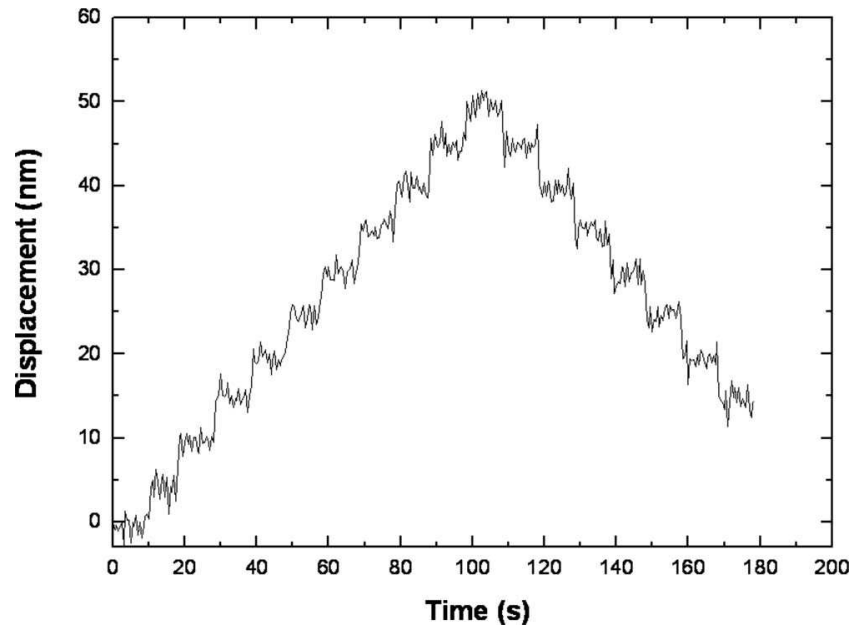

FIG. 3. Experimental result of a back and forth displacement by steps of 5 $\mathrm{nm}$ length. Position steps of $5 \mathrm{~nm}$ could easily be observed. The noise level is about $1.8 \mathrm{~nm}$.

to the phase of the $4 f$ harmonic component. As $S_{H 4}$ is divided by 8 in frequency, Eq. (10) becomes

$$
\Delta x=\frac{2 \lambda_{0} \Delta \varphi_{14 \mathrm{~Hz}}}{\pi n},
$$

where $\Delta \varphi_{14 \mathrm{~Hz}}$ is the phase of $S_{H 4}$.

The wavelength of our laser source (Renishaw ML10) has been calibrated under vacuum with regard to a national standard reference, and its value is $632.990577 \mathrm{~nm}$ with a relative uncertainty of $7.9 \times 10^{-8}$. The minimal phase shift possible with our digital signal generator is $0.01^{\circ}$ (174 $\mu \mathrm{rad})$. According to Eq. (11), the minimal theoretical step of displacement is $70 \mathrm{pm}$. So high level of accuracy can only be reached with a controlled surrounding environment. ${ }^{19,20}$ For this purpose, a weather station is installed to measure the room temperature (PT100, $1 \sigma$ $=0.01{ }^{\circ} \mathrm{C}$ ), pressure (Paroscientific, $\left.1 \sigma=3 \mathrm{~Pa}\right)$, and humid ity content (HygroM4, $1 \sigma=1 \%)$. Furthermore, our experimental system is mounted on a free mechanical vibration table and the polarimetric interferometer is sealed in a box with optical windows for the laser beams.

For the purpose of demonstration, phase shifts of as low as $0.71^{\circ}$ on the signal $S_{2}$ have been made. This corresponds to a theoretical displacement step value of $5 \mathrm{~nm}$. The phase shifts are sent with a periodicity of $10 \mathrm{~s}$. The phase shifts of $S_{H 4}$ relative to $S_{1}$ are simultaneously measured by the phase meter and converted in dimensional scale using Eq. (11). An example of back and forth displacements by steps of $5 \mathrm{~nm}$ is reported in Fig. 3. Position steps of $5 \mathrm{~nm}$ could easily be observed. The noise level is about $1.8 \mathrm{~nm}$. Position steps smaller than $5 \mathrm{~nm}$ can be achieved with the digital signal generator. As the phase resolution of the signal generator is about $0.01^{\circ}$, steps of as small as tens of picometers could be reached. To observe such small steps, the signal to noise ratio of the measurement board has to be improved. For this purpose, we plan to increase the polarization state modulation frequency by using Pockels cells instead of a rotating motor. This would permit to improve the signal to noise ratio and so, to achieve subnanometric steps. 


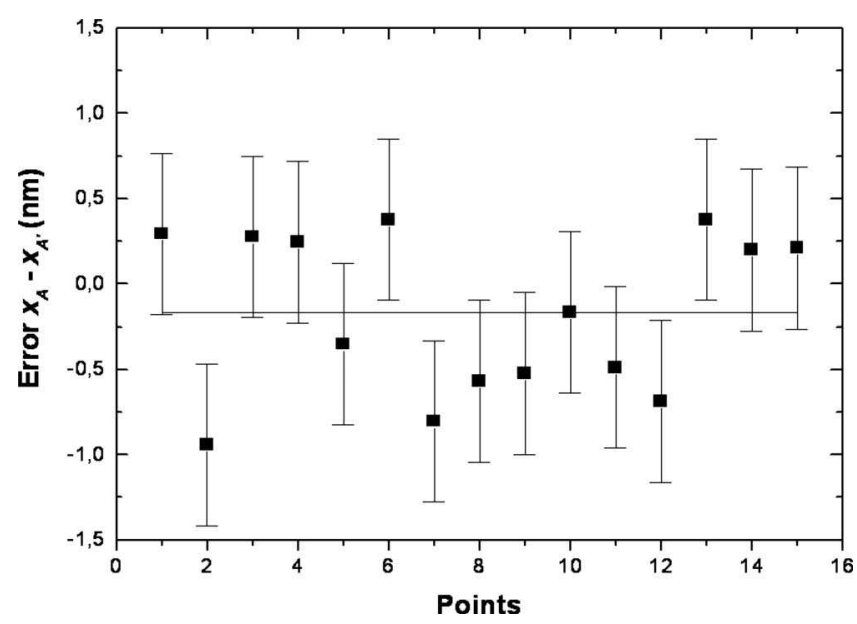

FIG. 4. Repeatability of the system. A displacement of $1 \mu \mathrm{m}$ with $50 \mathrm{~nm}$ steps has been repeated 15 times under similar conditions. We note $x_{A}$ and $x_{A}^{\prime}$, respectively, the first and last position values; the repeatability is defined as the standard deviation of the positioning errors $\left(x_{A}-x_{A}^{\prime}\right)$. The arithmetic mean value is equal to $-0.17 \mathrm{~nm}$ and the standard deviation $(1 \sigma)$ is equal to $0.47 \mathrm{~nm}$.
To estimate the repeatability of our displacement system, we repeat 15 times a $1 \mu \mathrm{m}$ displacement by steps of $50 \mathrm{~nm}$ under similar conditions. If we note $x_{A}$ and $x_{A}^{\prime}$, respectively, the first and last position values, the repeatability is defined as the standard deviation of the positioning errors $\left(x_{A}-x_{A}^{\prime}\right)$. The result is shown in Fig. 4. The arithmetic mean value is equal to $-170 \mathrm{pm}$ and the standard deviation $(1 \sigma)$ is equal to $470 \mathrm{pm}$.

To analyze the resolution of the system, the target mirror is wedged and the loop control is locked, i.e., the signal $S_{H 4}$ is phase locked on the signal $S_{2}$. The signal to noise ratio is recorded over about $350 \mathrm{~s}$. Figure 5 represents the temporal distribution of the signal to noise ratio converted in nanometers (upper graph) and the corresponding statistical result (lower graph). The histogram is plotted with a normal distribution overlay. A noise level at $1 \sigma$ of $=1.8 \mathrm{~nm}$ is found. A linear regression on the temporal distribution has been calculated and represented in the figure by the straight line. It is quite superimposed onto the zero line because the slope is equal to $-8.9 \times 10^{-5} \mathrm{~nm} / \mathrm{s}$. We can consider that there is no significant drift.

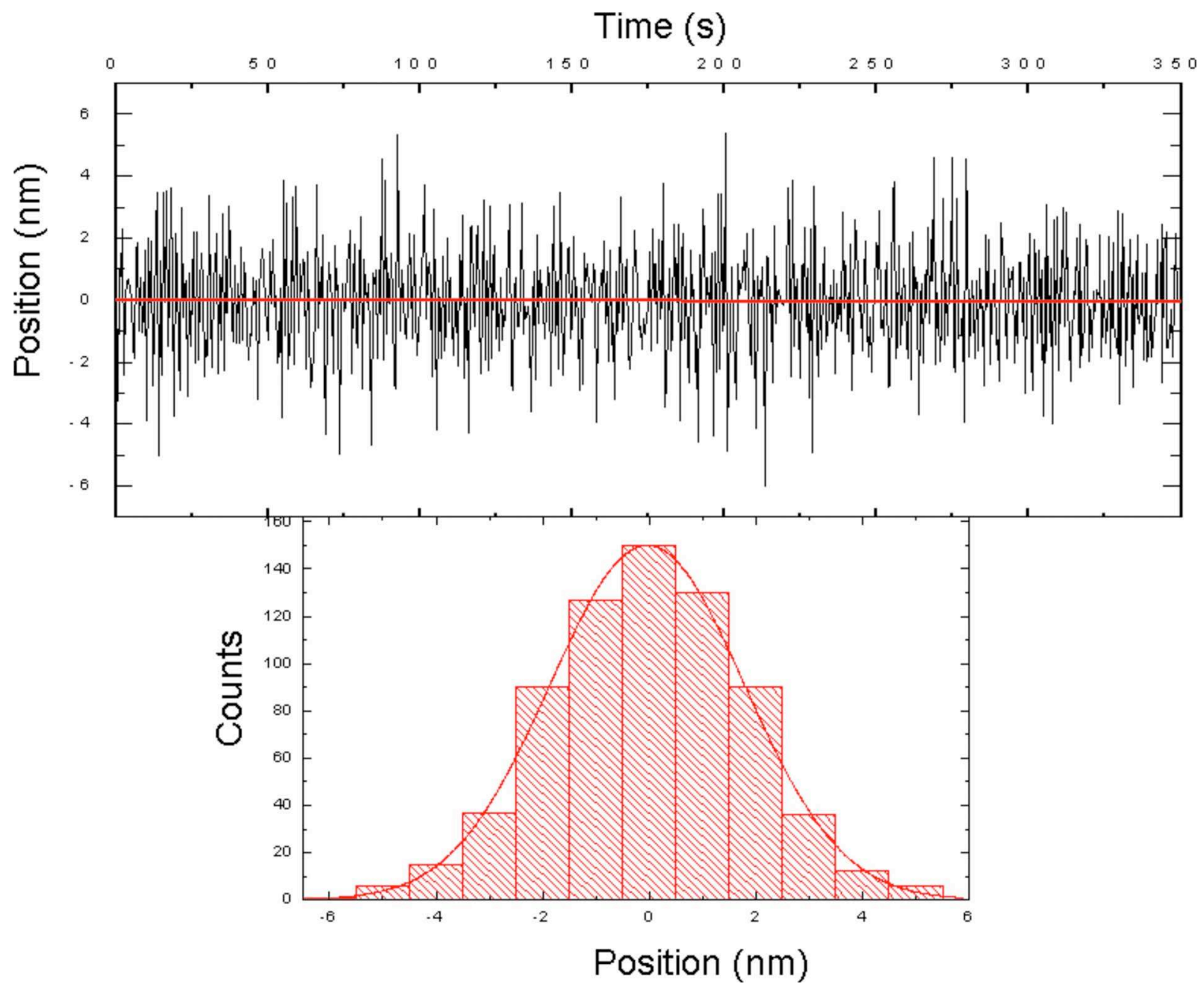

FIG. 5. (Color online) Temporal distribution of the signal to noise ratio converted in nanometers (upper graph) and the corresponding statistical result (lower graph). The signal to noise ratio is recorded over about $350 \mathrm{~s}$. The histogram is plotted with a normal distribution overlay. A standard deviation of $1 \sigma=1.8 \mathrm{~nm}$ is found. With the use of a linear regression analysis on the temporal distribution, a long term drift (straight line) equal to $-8.9 \times 10^{-5} \mathrm{~nm} / \mathrm{s}$ is calculated. 


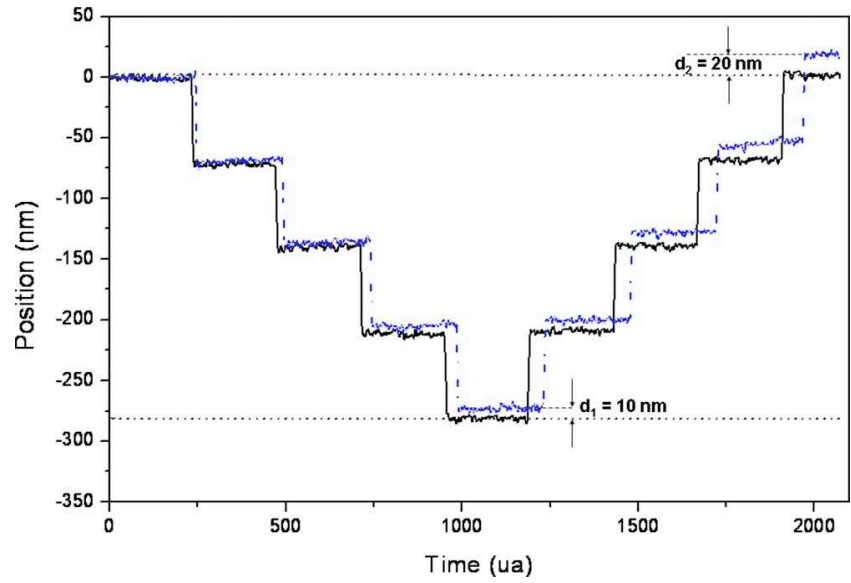

FIG. 6. (Color online) Position step of $70 \mathrm{~nm}$ for different residual ellipticity values. (a) Straight curve $\varepsilon=0^{\circ}$. (b) Dashed curve $\varepsilon=10^{\circ}$. The dashed curve has been horizontally shifted in order to improve readability of the figure. Error due to residual ellipticity appears clearly.

\section{DISCUSSIONS}

In this section, we discuss some errors that affect the uncertainty of the displacement measurements made with our system. One of these errors is specific to our method, i.e., the residual ellipticity of the polarization state of the laser beam. It is widely discussed hereafter. Other sources of errors, most common to displacement measurements made by optical interferometry, are also estimated and references are given for further details. We also discuss the main parameter limiting the resolution of our prototype and the solution we plan to set up in order to improve the signal to noise ratio.

\section{A. Influence of a residual ellipticity on displacement measurement}

In order to estimate the influence on the displacement measurement of a residual ellipticity on the polarization state of the laser beam, the quarter-wave plate is slightly misadjusted (rotated). Then phase shifts of $10^{\circ}$ are done on the $S_{2}$ signal corresponding to position steps of $70 \mathrm{~nm}$. Figure 6 shows the results of two displacements, respectively, with a perfectly linear polarization state $\left(\varepsilon=0^{\circ}\right)$ and with an ellipticity of $\varepsilon=10^{\circ}$, which corresponds to a worst case adjustment possible, taking into account the efficiency of the optical mounts. In this first experiment, the bandpass filter is a second order Rauch filter.

As the rejection ratio between the amplitudes of the $2 f$ and $4 f$ harmonic components is linked to the value of the ellipticity, we developed a theoretical model to simulate the relation between the error on positioning due to the residual ellipticity and this rejection ratio. The simulation takes into account the influence of the second harmonic on the phase of the fourth harmonic with the amplitude and the phase parameters.

Experimental measurements of the ratio are made, thanks to a spectrum analyzer (SR785, Stanford). We measure a rejection ratio after the filter of about $35 \mathrm{~dB}$ for $\varepsilon=0^{\circ}$ and $10 \mathrm{~dB}$ for $\varepsilon=10^{\circ}$. We put these values in our model and found that for $\varepsilon=0^{\circ}$ the theoretical result of the positioning error is around $1 \mathrm{~nm}$. For $\varepsilon=10^{\circ}$, the expected

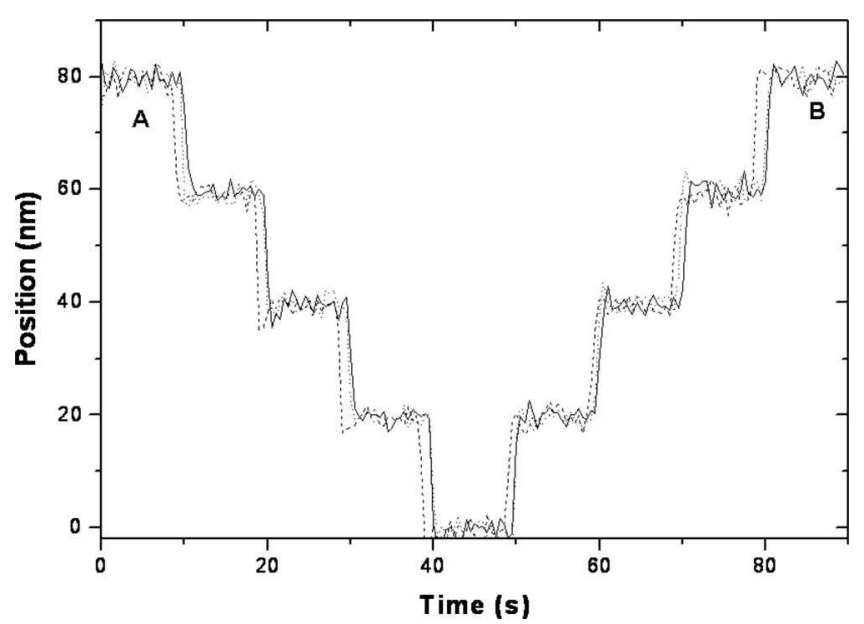

FIG. 7. Position step of $20 \mathrm{~nm}$ corresponding to different ellipticity values. (a) Straight curve $\varepsilon=0$. (b) Dotted curve $\varepsilon=5^{\circ}$. (c) Dashed curve $\varepsilon=10^{\circ}$. The effect of a residual ellipticity of the laser beam polarization state is canceled out by the effect of the filtering of the second harmonic.

positioning error is around $10 \mathrm{~nm}$, which is in good agreement with the experimental measurement seen on the dashed curve of Fig. 6. To improve our system, we modified the electronic board. The Rauch filter is replaced by an eighth order continuous-time active filter, allowing a rejection ratio of $40 \mathrm{~dB}$ even with a residual ellipticity of $10^{\circ}$. To validate the new system, back and forth phase shifts equal to $2.84^{\circ}$ $(\sim 20 \mathrm{~nm})$ are made on $S_{2}$ with residual ellipticity values of, respectively, $\varepsilon=0^{\circ}, \varepsilon=5^{\circ}$, and $\varepsilon=10^{\circ}$. The results are shown in Fig. 7. The effect of a residual ellipticity of the laser beam polarization state is canceled out by the effect of the filtering of the second harmonic. In Table I, we report the positioning error between the first point (A) and the destination point (B) for the different values of $\varepsilon$.

Taking into account that the resolution of the measurement board is $1.8 \mathrm{~nm}$, we can consider that the effect of a residual ellipticity less than $10^{\circ}$ on the polarization state of the laser beam could be negligible on the error budget of the displacement measurements with our apparatus.

\section{B. Other sources of error and forthcoming works}

All the common sources of errors such as diffraction, cosine error, and laser frequency stability have to be also taken into account for accurate displacement measurements with our system. Systematic and statistical errors in interferometry are well known and have been discussed in length by

TABLE I. Position step errors for different ellipticity values. Position A and position B are, respectively, the starting and final positions illustrated in Fig. 7, averaged over 20 measurements points. The error is the difference (A $-\mathrm{B}$ ). Taking into account that the resolution of the measurement board is 1.8 $\mathrm{nm}$, we can consider that the effect of a residual ellipticity less than $10^{\circ}$ on the polarization state of the laser beam could be negligible on the error budget of the displacement measurements with our apparatus.

\begin{tabular}{lccc}
\hline \hline & $\varepsilon=0^{\circ}$ & $\varepsilon=5^{\circ}$ & $\varepsilon=10^{\circ}$ \\
\hline Position A (nm) & 79.51 & 79.40 & 79.14 \\
Position B (nm) & 79.81 & 79.81 & 79.75 \\
Error A-B (nm) & -0.30 & -0.41 & -0.61 \\
\hline \hline
\end{tabular}


several authors. ${ }^{20,21}$ Some systematic errors as those caused from plane mirror misalignment (parallel, orthogonal, and cosine errors), thermal expansion, and flatness of the mirror are negligible. The total uncertainty includes contributions from the misalignment between the beams, the frequency stability of the lasers, the refractive index of air, and the frequency stability of the Doppler shift. These uncertainties can be added in quadrature to produce an estimate for the uncertainty on the measurement.

The signal to noise ratio of our interferometer is limited by the spinning motor used in the polarimeter head. One reason is due to its rotation speed, which is about $20-30 \mathrm{~Hz}$, limiting the bandwidth of our lock-in loops to tens of hertz. The second reason is due to the mechanical defects of the spinning motor that add phase disturbances on the optical signal at the output of the polarimeter. To improve our system, we plan to use an electro-optic phase modulator instead of a rotating quarter-wave plate. Such device could permit a phase modulation at some megahertz level without any mechanical rotation permitting to reach picometer resolution and repeatability.

\section{ACKNOWLEDGMENTS}

The authors are grateful toward F. Mourgues for his efficiency in the realization of the interferometer mechanical parts.
${ }^{1}$ I. Misumi, S. Gonda, T. Kurosawa, and K. Takamasu, Meas. Sci. Technol. 14, 463 (2003).

${ }^{2}$ K. Dirscherl and K. R. Koops, Proc. SPIE 5190, 173 (2003).

${ }^{3}$ T. Ruijl, Ph. D. thesis, TUD, 2001.

${ }^{4}$ The International Roadmap for Semiconductors 2003 edition metrology (http://public.itrs.net), December 2003.

${ }^{5}$ S. Topcu, L. Chassagne, D. Haddad, and Y. Alayli, Rev. Sci. Instrum. 74, 4876 (2003).

${ }^{6}$ S. Topcu, L. Chassagne, Y. Alayli, and P. Juncar, Opt. Commun. 247, 133 (2005).

${ }^{7}$ L. Chassagne, S. Topcu, Y. Alayli, and P. Juncar, Meas. Sci. Technol. 16, 1771 (2005).

${ }^{8}$ A. Sinno, P. Ruaux, L. Chassagne, S. Topçu, and Y. Alayli, Rev. Sci. Instrum. 78, 095107 (2007).

${ }^{9}$ L. Chassagne, M. Wakim, S. Xu, S. Topçu, P. Ruaux, P. Juncar, and Y. Alayli, Meas. Sci. Technol. 18, 3267 (2007).

${ }^{10}$ R. C. Jones, J. Opt. Soc. Am. 31, 488 (1941)

${ }^{11}$ R. C. Jones, J. Opt. Soc. Am. 31, 500 (1941).

${ }^{12}$ R. C. Jones, J. Opt. Soc. Am. 31, 486 (1942).

${ }^{13}$ R. M. A. Azzam and N. M. Bashara, Ellipsometry and Polarized Light, edited by Elsevier (North-Holland, Amsterdam, 1989).

${ }^{14}$ D. Goldstein, Polarized Light: The Poincaré Sphere (Dekker, New York, 2003).

${ }^{15}$ D. Goldstein, Polarized Light: Methods of Measuring the Stokes Polarization Parameters (Dekker, New York, 2003).

${ }^{16}$ P. A. Williams, Appl. Opt. 38, 6508 (1999).

${ }^{17}$ W. G. Schweitzer, Jr., E. G. Kessler, Jr., R. D. Deslattes, H. P. Layer, and J. R. Whetstone, Appl. Opt. 12, 2927 (1973).

${ }^{18}$ T. M. Niebauer, J. E. Faller, H. M. Godwin, J. L. Hall, and R. L. Barger, Appl. Opt. 27, 1285 (1988).

${ }^{19}$ K. P. Birch and M. J. Downs, Metrologia 30, 155 (1993).

${ }^{20}$ N. Bobroff, Meas. Sci. Technol. 4, 907 (1993).

${ }^{21}$ R. Castell, W. Demtroder, A. Fisher, R. Kullmer, H. Weickenmeier, and K. Wickert, Appl. Opt. 38, 1 (1985). 